
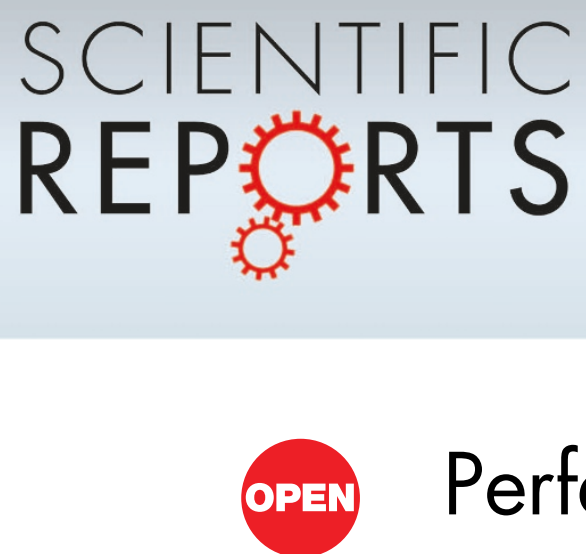

\title{
Perfect anti-reflection from first principles
}

\author{
Kyoung-Ho Kim \& Q-Han Park
}

SUBJECT AREAS:

OPTICAL PHYSICS

OPTICAL MATERIALS AND

STRUCTURES

APPLIED PHYSICS

METAMATERIALS

Received

24 August 2012

Accepted

3 December 2012

Published

14 January 2013

Correspondence and requests for materials should be addressed to Q.P. (qpark@korea. ac.kr)
Department of Physics, Korea University, Seoul 136-701, Korea.

Reducing unwanted reflections through impedance matching, called anti-reflection, has long been an important challenge in optics and electrical engineering. Beyond trial and error optimization, however, a systematic way to realize anti-reflection is still absent. Here, we report the discovery of an analytic solution to this long standing problem. For electromagnetic waves, we find the graded permittivity and permeability that completely remove any given impedance mismatch. We demonstrate that perfect broadband anti-reflection is possible when a dispersive, graded refractive index medium is used for the impedance-matching layer. We also present a design rule for the ultra-thin anti-reflection coating which we confirm experimentally by showing the anti-reflection behavior of an exemplary $\lambda / 25$-thick coating made of metamaterials. This work opens a new path to anti-reflection applications in optoelectronic device, transmission line and stealth technologies.

wave is partially reflected when it enters a different medium. A fraction of the light or the electromagnetic (EM) waves in general, is reflected due to the mismatch of impedance caused by the abrupt change of refractive index. An anti-reflection coating of a quarter-wave optical thickness is a well-known method for removing reflections of one specific wavelength, while more complex structures, such as multilayer coatings or moth-eye-type graded index structures, are used for broadband anti-reflection applications ${ }^{1-3}$. Such a quarterwave coating is severely limited in application due to the strict condition on the thickness and refractive index of a coating material whereas complex structures for broadband anti-reflection have no systematic design rules due to the lack of analytic solutions. The reflection of light passing through a medium with a graded refractive index was first studied in 1879 by Rayleigh, who analytically proved that reflection becomes significantly reduced if the medium, whose graded index is a function of the inverse square of the thickness, has an overall thickness greater than the wavelength of light ${ }^{4}$. Moth eyes covered in arrays of tapered pillars of subwavelength size also act as a graded refractive index medium reducing reflection ${ }^{5}$. The moth eye anti-reflection scheme has been recently applied to various optoelectronic devices including light-emitting diodes ${ }^{6}$, displays ${ }^{7}$, photovoltaic solar cells $s^{8}$ and micro sun sensor' ${ }^{9}$. However, except in some special cases such as the Rayleigh case ${ }^{4,10}$, analytic solutions are not known for a graded index medium, thus an explicit knowledge of the wave impedance and impedance mismatches causing reflection is lacking. Subsequently, designers of anti-reflection coatings, utilizing either a motheye structure or a multilayer coating, rely mostly on a numerical trial-and-error procedure to find optimized anti-reflection structures ${ }^{11,12}$. The lack of first principles in anti-reflection theory is more troublesome in the lower frequency $\mathrm{THz}$ and the microwave regime, where conventional wisdom, which holds that the coating thickness be greater than the half-wavelength ${ }^{13,14}$, severely restricts applications. The same difficulty exists in the antireflection technology for transmission lines using various types of tapered impedance matching transformers ${ }^{15,16}$.

In this letter, we propose a new analytic approach to the anti-reflection problem that relates the graded refractive index directly to the wave impedance. Unlike the conventional approach, we are able to find explicitly graded permittivity and permeability profiles that produce any desired locally varying wave impedances. This approach enables a systematic realization of broadband and ultrathin anti-reflection layers by determining explicit conditions for the impedance matching. In particular, a perfect broadband anti-reflection structure is shown to be possible when a dispersive, graded index medium is used for the impedance-matching layer. We experimentally demonstrate the anti-reflection behavior of graded index profiles designed according to our impedance matching method. The ultrathin anti-reflection behavior is observed with an exemplary $\lambda / 25$-thick coating made of metamaterials, the thinnest anti-reflection coating ever achieved.

\section{Results}

First principles method. Conventional anti-reflection technology relies on trial and error optimization of refractive indices of coating layers to reduce the impedance mismatch. Here, we show that there exists a systematic method of finding inhomogeneous coating layers that reproduce any given profile of wave impedance, in particular a profile that removes the impedance mismatch completely. Consider the Maxwell's 
equation for one-dimensional plane wave propagating along the $x$ direction,
(a) $\frac{\partial E_{y}}{\partial x}=-\mu(x) \frac{\partial H_{z}}{\partial t}$
(b) $\frac{\partial H_{z}}{\partial x}=-\varepsilon(x) \frac{\partial E_{y}}{\partial t}$.

We assume that the permittivity $\varepsilon(x)$ and the permeability $\mu(x)$ of an inhomogeneous medium vary along the $x$-direction. For a monochromatic wave, we may express the electric and the magnetic field components by

$$
E_{y}=P(x) e^{i Q(x)-i \omega t}, H_{z}=Y(x) E_{y}
$$

where the amplitude $P(x)$ and phase $Q(x)$ are real functions of $x$ and $Y(x)=H_{z} / E_{y}$ is the wave admittance. The Maxwell's equation then reduces to

$$
\text { (a) } i \omega \mu Y E_{y}=E_{y, x} \quad \text { (b) } i \omega \varepsilon E_{y}=Y_{, x} E_{y}+Y E_{y, x}
$$

where a subscript comma followed by the index $x$ indicates partial differentiation with respect to $x$. By eliminating $E_{y}$ from equation (3), we find a relation between the material's permittivity and permeability and the wave admittance,

$$
\varepsilon=\frac{1}{i \omega} Y_{, x}+\mu Y^{2}
$$

which is one of the main result of the present work. This tells us that material indices $\varepsilon$ and $\mu$ can be simply chosen from the above relation to reproduce any desired wave admittance $Y$. Here, for simplicity, we restrict to the case of a lossless medium for which $\varepsilon(x)$ and $\mu(x)$ are real. Then, denoting the real and imaginary parts of admittance by $Y=Y_{R}+i Y_{I}$ and evaluating the real and imaginary parts of equation (4) separately, we obtain

$$
\mu=\frac{Y_{R, x}}{2 \omega Y_{I} Y_{R}}, \quad \varepsilon=\frac{Y_{I, x}}{\omega}+\frac{Y_{R, x}}{2 \omega}\left(\frac{Y_{R}}{Y_{I}}-\frac{Y_{I}}{Y_{R}}\right) .
$$

If we further restrict to a non-magnetic medium possessing a vacuum permeability $\left(\mu=\mu_{0}\right)$, we have

$Y_{I}=\frac{Y_{R, x}}{2 \omega \mu_{0} Y_{R}}, \quad \varepsilon=\frac{1}{2 \omega^{2} \mu_{0}}\left(\frac{Y_{R, x}}{Y_{R}}\right)_{, x}+\mu_{0} Y_{R}^{2}-\frac{1}{4 \omega^{2} \mu_{0}}\left(\frac{Y_{R, x}}{Y_{R}}\right)^{2}$.

If the medium is lossless, we have a conserved intensity, i.e. a conserved time-averaged Poynting vector. This allows us to rewrite the real part of admittance in terms of the electric field amplitude $P(x)$ and the conserved intensity $S$ such that

$$
Y_{R}=\frac{2 S}{P^{2}} \text {. }
$$

This brings the permittivity in (6) to the expression,

$$
\varepsilon(x)=\frac{4 \mu_{0} S^{2}}{P^{4}}-\frac{1}{\omega^{2} \mu_{0}} \frac{P_{, x x}}{P},
$$

and also makes $Q(x)$ and $Y(x)$ to be expressed by

$$
Q(x)=\int \frac{2 \omega \mu_{0} S}{P^{2}} d x, Y(x)=\frac{P_{, x}}{i \omega \mu_{0} P}+\frac{2 S}{P^{2}} .
$$

The wave impedance, as the inverse of admittance, can be also expressed in terms of $P(x)$. For counter propagating waves, we find the wave impedance,

$$
Z_{ \pm}(x)=\frac{E_{y}}{H_{z}}=\left[ \pm \frac{P_{, x}}{i \omega \mu_{0} P}+\frac{2 S}{P^{2}}\right]^{-1}
$$

where the subscript ' \pm ' indicates the sense of propagation direction with the minus sign corresponding to the counter propagating wave (change $Q(x)$ by $-Q(x)$ ). Thus the electric field amplitude $P(x)$ plays the role of a potential from which other physical quantities are derived. This provides us an elegant way to design graded index profiles with specific impedance properties in mind.

In order to find conditions for the graded index anti-reflection, we consider light entering normally into a medium with refractive index $n_{2}$ (region II) from a medium with index $n_{1}$ (region I). In the presence of a non-magnetic coating layer of thickness $d$ with a graded index (region $\mathrm{G}$ ), we assume electric field profiles in each region as follows;

$$
\begin{aligned}
E_{y}^{I} & =e^{i n_{1} k_{0} x}+r e^{-i n_{1} k_{0} x}, \\
E_{y}^{G} & =A P(x) e^{i Q(x)}+B P(x) e^{-i Q(x)}, \\
E_{y}^{I I} & =t e^{i n_{2} k_{0} x} .
\end{aligned}
$$

Matching boundaries at each interfaces and solving for the undetermined coefficients, we obtain the reflection coefficient,

$$
r=\frac{\left[\frac{n_{1}-Z_{0} Z_{+}^{-1}(0)}{n_{1}+Z_{0} Z_{+}^{-1}(0)}\right]-\left[\frac{n_{1}+Z_{0} Z_{-}^{-1}(0)}{n_{1}+Z_{0} Z_{+}^{-1}(0)}\right]\left[\frac{n_{2}-Z_{0} Z_{+}^{-1}(d)}{n_{2}+Z_{0} Z_{-}^{-1}(d)}\right] \exp (2 i[Q(d)-Q(0)])}{1-\left[\frac{n_{1}-Z_{0} Z_{-}^{-1}(0)}{n_{1}+Z_{0} Z_{+}^{-1}(0)}\right]\left[\frac{n_{2}-Z_{0} Z_{+}^{-1}(d)}{n_{2}+Z_{0} Z_{-}^{-1}(d)}\right] \exp (2 i[Q(d)-Q(0)])},(12)
$$

a

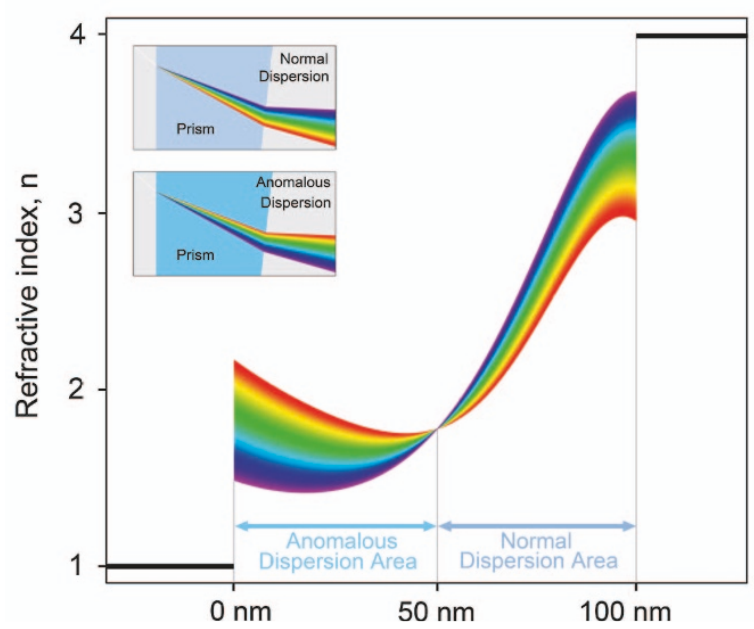

b

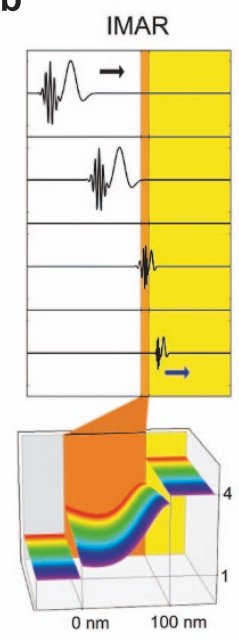

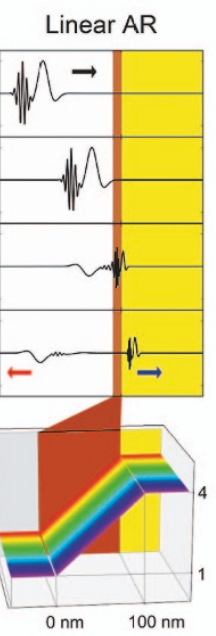

Without AR

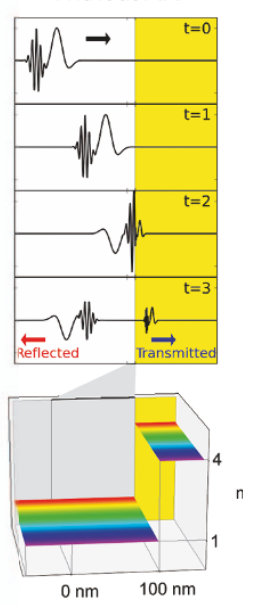

Figure 1 Perfect broadband anti-reflection. (a) Graded refractive index profile enabling a complete impedance matching for all frequencies. Dispersion of the graded index profile is expressed by rainbow colors where each color represents the corresponding frequency of visible light. Anomalous and normal dispersion regions are shown. (b) Reflection and transmission of a short time pulse entering from air $(n=1)$ into a medium $(n=4)$ with an impedance-matched anti-reflection (IMAR) layer, linearly graded AR layer and no AR layer. The linear AR layer reflects the low frequency components while the IMAR layer shows no reflection at all (see the supporting movies for the pulse propagation). 
where $Z_{0}$ is the impedance of vacuum. Vanishing reflection $(r=0)$ occurs if

$$
\begin{aligned}
& n_{1}=Z_{0} Z_{+}^{-1}(0)=\frac{Z_{0} P_{, x}(0)}{i \omega \mu_{0} P(0)}+\frac{2 Z_{0} S}{P(0)^{2}}, \\
& n_{2}=Z_{0} Z_{+}^{-1}(d)=\frac{Z_{0} P_{, x}(d)}{i \omega \mu_{0} P(d)}+\frac{2 Z_{0} S}{P(d)^{2}},
\end{aligned}
$$

or

$$
P_{, x}(0)=0=P_{, x}(d), \quad P(0)^{2}=\frac{2 Z_{0} S}{n_{1}}, \quad P(d)^{2}=\frac{2 Z_{0} S}{n_{2}} .
$$

The permittivity $\varepsilon(x)$, determined through equation (8) with a chosen $P(x)$ satisfying the boundary condition in equation (14), admits a complete impedance matching valid for all frequencies. In other words, we now achieve an achromatic perfect anti-reflection!

Perfect broadband anti-reflection. Perfect anti-reflection is demonstrated in Fig. 1 with an exemplary choice of $P(x)$

$P(x)=\sqrt{2 \mu_{0} c S}\left[\left(n_{1}^{-1 / 2}-n_{2}^{-1 / 2}\right)\left(2 x^{3} / d^{3}-3 x^{2} / d^{2}\right)+n_{1}^{-1 / 2}\right]$,

where $c$ is the speed of light in vacuum. We point out that the impedance matching boundary condition with two distinct nondispersive dielectric media $\left(n_{1} \neq n_{2}\right)$ inevitably demands the inhomogeneous medium to possess both the normal dispersion region and the anomalous dispersion region as illustrated in Fig. 1. The graded refractive index profile of thickness $100 \mathrm{~nm}$ is displayed in Fig. 1a for wavelengths ranging from $400 \mathrm{~nm}$ to $700 \mathrm{~nm}$. There is no restriction on the layer thickness and $100 \mathrm{~nm}$ is chosen only for convenience. Note that, contrary to expectation, impedance matching requires the graded index profile to be discontinuous at each interface $(x=0 \mathrm{~nm}$ and $x=100 \mathrm{~nm}$ ). To demonstrate the perfectly anti-reflecting behavior, we send a short pulse composed of broadband frequency components into a dielectric medium prepared in three different ways: i) without any layer, ii) with a non-dispersive linear grading layer, and iii) with our perfect impedance matching layer. In Fig. 1b, the reflecting behaviors of a pulse in these three cases, which are calculated numerically using the Finite Difference Time Domain (FDTD) method, are shown. Despite a sizable reduction in reflection, the linear grading case fails to reduce the reflection of lower frequency components. In contrast, the impedance matching layer shows no reflection at all for any frequency component, thus demonstrating a perfect broadband anti-reflection behavior (Supplementary movie 1-3). The frequency-dependent terms for the permittivity and the impedance in equations (8) and (10) become negligible if the wavelength is much shorter than the layer thickness. Subsequently, impedance matching for a thick layer requires only the grade index to be continuous at the interface. This explains why nature-provided graded index structures, such as earth's atmosphere, exhibit anti-reflection behavior for sunlight. In order to realize perfect anti-reflection, we need a transparent medium exhibiting anomalous dispersion, such as the one used for superluminal light propagation ${ }^{17}$ which is quite difficult to apply for our purpose. Moreover, anomalous dispersion is generally accompanied with a strong absorption. Therefore, making a practical, thin, perfect anti-reflection layer poses important future challenges.

Graded refractive index anti-reflection. If the layer consists of a non-dispersive medium that matches impedance only for a particular frequency, it acts as a monochromatic anti-reflection layer without the thickness restriction. This is in sharp contrast to the conventional quarter wave anti-reflection coating where the coating thickness should be one quarter of the wavelength in the coating layer with a refractive index $\left(n_{1} n_{2}\right)^{1 / 2}$. To justify the validity of our impedance matching method for the graded index anti-reflection, we conducted an experiment on microwave

propagation through a rectangular metallic waveguide and measured the reflectance of samples with various anti-reflection structures. The waveguide is prepared to support only the TE10 mode in the frequency range of $1.8 \mathrm{GHz}$ to $2.2 \mathrm{GHz}$. Due to the confinement, the TE10 mode propagates with a reduced wave number $\beta$. Thus it simulates a plane wave propagating with a wave number $k$ through a medium with the effective refractive index $n_{\text {eff }}=$ $\beta / k$.

Figure 2 shows a schematic diagram of our waveguide setup loaded by Teflon slab samples with anti-reflection structures attached. The effective refractive index of the air-filled waveguide is 0.73 while that of the Teflon-filled waveguide is 1.24 at a frequency of $2 \mathrm{GHz}$. Antireflection structures are made of Teflon and act effectively as a graded impedance matching layer (Fig. 2a) and a quarter-wave layer (Fig. 2b). They are designed to possess anti-reflection properties at a frequency of $2 \mathrm{GHz}$ utilizing the effective refractive index of partially filled waveguides (Supplementary). The quarter-wave structure is $4 \mathrm{~cm}$ thick and possesses an effective refractive index 0.95 at $2 \mathrm{GHz}$ to meet the quarter-wave anti-reflection condition. The graded structure, with a freely chosen thickness of $3 \mathrm{~cm}$, has an inverted moth-eye type shape with the effective refractive index profile shown in Fig. 2a. We placed anti-reflection structures symmetrically on both sides of a Teflon slab to avoid the reflection from the uncoated face. Experimental results are presented in Fig. 2c, which are in good agreement with theoretical predictions of anti-reflection at $2 \mathrm{GHz}$. Both the graded structure and the quarter-wave structure show near zero reflectance at $2 \mathrm{GHz}$, thereby confirming our graded

a

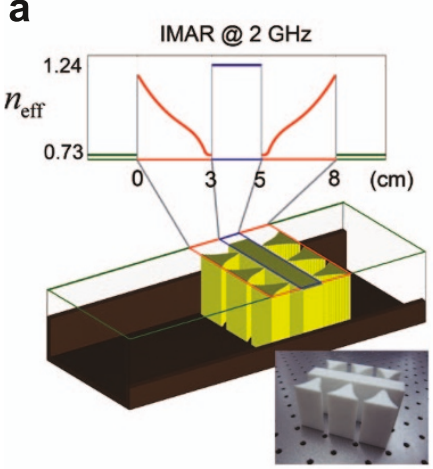

b

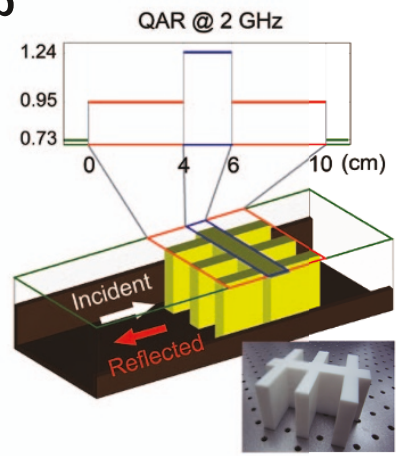

C

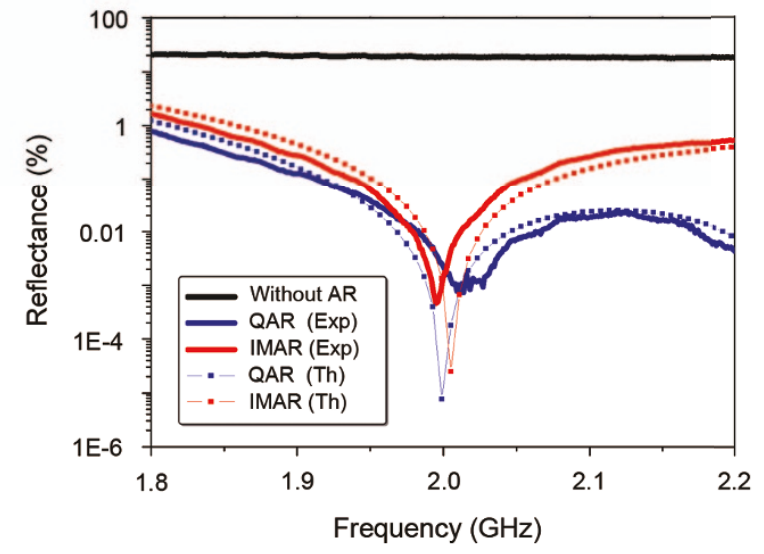

Figure $2 \mid$ Graded index anti-reflection. (a \& b) Experimental setup for the microwave measurement of anti-reflection using (a) an inverted motheye structure realizing the impedance-matched anti-reflection (IMAR) and (b) a partially filled slab structure realizing the quarter-wave antireflection (QAR). Each structure is designed to possess effective refractive indices for anti-reflection inside a waveguide. (c) Measured reflectance (solid lines) for IMAR (red) and QAR (blue) structures in comparison with theoretical predictions (dotted lines). 
impedance matching approach. It is remarkable that, as the thickness of a graded index profile becomes smaller than that of the quarterwave, the discontinuity of refractive indices at each interface becomes more pronounced making an inverted moth-eye-type graded structure.

Ultra-thin anti-reflection. As there is no thickness restriction for anti-reflection, we may ask what happens if the thickness of a layer becomes extremely small. The graded permittivity given in equations (8) and (15) takes both positive and negative values if the thickness $d$ is much smaller than the wavelength $\lambda$. This raises the possibility of ultrathin anti-reflection coatings using both positive and negative permittivity coating materials. To prove the possibility explicitly, we have constructed ultrathin anti-reflection layers using meta-

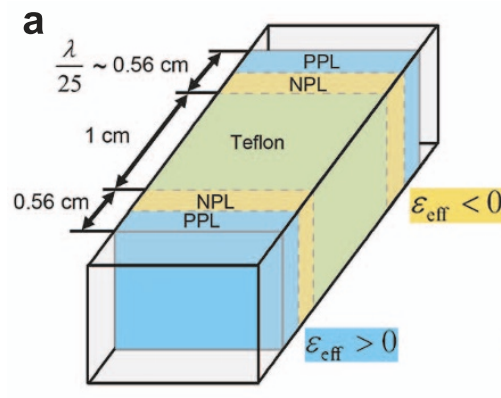

Negative Permittivity Layer (NPL)

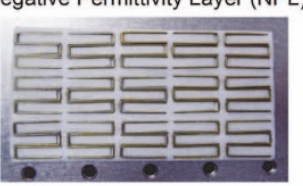

Positive Permittivity Layer (PPL)

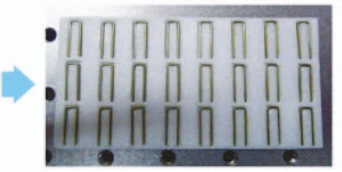

b

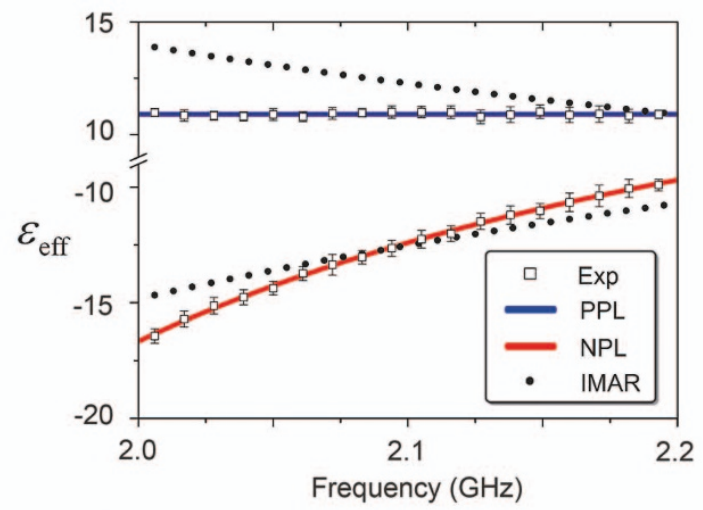

C

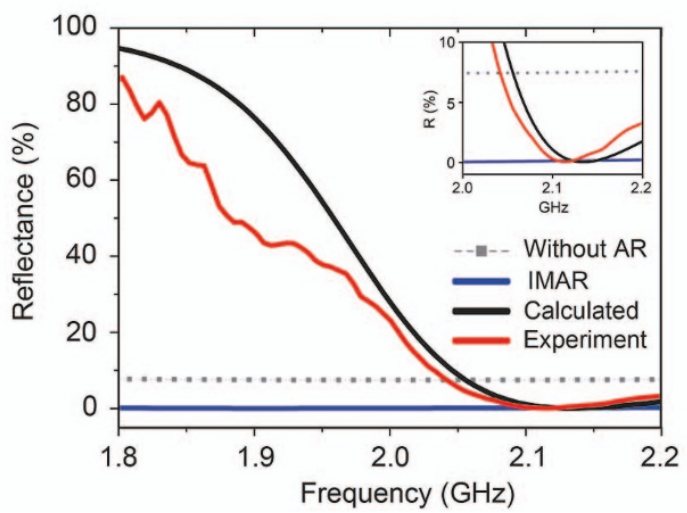

Figure 3 | Ultra-thin anti-reflection. (a) Schematic diagram of two-layer ultra-thin AR using a negative permittivity layer (NPL) and a positive permittivity layer (PPL) attached to a Teflon slab. Metamaterials NPL and PPL are shown in the pictures. (b) Measured permittivity of metamaterials (open squares with error bars). Solid lines are the Drude-Lorentz model fit and dotted lines present the permittivity of PPL and NPL predicted by the two layer approximation of IMAR. (c) Measured reflectance (red) and theoretical predictions using the measured permittivity (black) and using the two-layer approximation of IMAR (blue). Blue line shows that the approximated IMAR produces nearly perfect anti-reflection. materials and measured reflectances, with results described in Fig. 3. To avoid the technical difficulty of preparing a continuously varying permittivity, we approximate the permittivity profile by a double layer, such that two layers of equal thickness possess positive and negative permittivity with values obtained from evaluating the graded permittivity in equation (8) at the central location of each layer. As demonstrated in Fig. 3c, this two-layer approximation produces nearly perfect anti-reflection despite a crude approximation (blue line). Figure $3 a$ shows a schematic diagram for the ultra-thin anti-reflection setup. Positive and negative permittivity layers made of metamaterials are attached to the Teflon sample in a symmetric way. The overall thickness of an anti-reflection structure is $0.56 \mathrm{~cm}$, which amounts to one twenty-fifth of the wavelength $(\lambda /$ 25) at frequency 2.1 GHz. Metamaterials are made of U-shaped zinc coated iron staples embedded in a polystyrene foam slab. They are designed to yield the positive and negative permittivity values (Supplementary). Experimental results in Fig. $3 \mathrm{~b}$ show the measured permittivity having non-dispersive positive and dispersive negative behaviors which fit well with the Drude-Lorentz model. They are compared with the ideal cases of impedance matching anti-reflection (IMAR) layers whose dispersive permittivities are plotted as dotted lines (filled circles) in Fig. 3b. With this material characterization, the reflectance of a sample from Fig. 3a can be calculated theoretically using the transfer matrix method. The result is presented in Fig. $3 \mathrm{c}$ as a solid line (black) in the case of a metamaterial sample and a flat solid line (blue) in the ideal IMAR case. This theoretical prediction is in good agreement with the experimentally measured reflectance, which is also presented in Fig. 3c. The measured reflectance shows a dip at $2.11 \mathrm{GHz}$ with the minimum value of $0.06 \%$. This confirms the ultra-thin anti-reflection property with a layer whose thickness is six times smaller than that of a conventional quarter-wave coating. To our knowledge, this is the thinnest anti-reflection layer so far constructed especially when compared with other metamaterialbased anti-reflection layers ${ }^{18}$. But this record can be easily broken with higher permittivity metamaterials.

\section{Discussion}

Here, we found explicitly graded permittivity and permeability profiles that produce any desired locally varying wave impedances. We have demonstrated ultra-thin anti-reflections and provided a design rule for the perfect broadband anti-reflection. Our paper not only presents a solution to the fundamental problem of graded index antireflection, first started by Rayleigh in 1879 , but more importantly it raises new opportunities in nanophotonics research. In particular, our work clarifies the working principle for the ultra-thin antireflection using plasmonic metal layers or metamaterials. Our first principles method can be easily applied to the transmission line system in microwave technology and also resolve the impedance matching problem of transmission lines.

\section{Methods}

For the microwave experiment, we used the Network Analyzer HP8719C, Coaxial-toWaveguide Adaptor and the standard rectangular waveguide WR430 $(10.92 \mathrm{~cm} \times$ $5.46 \mathrm{~cm}$ ) to generate the TE10 mode and measure the transmission and reflection within the frequency range $1.8 \mathrm{GHz}$ to $2.2 \mathrm{GHz}$. To remove systematic errors, we calibrated the connected components using the Thru-Reflect-Line calibration ${ }^{16}$. Graded index samples are made of Teflon having a refractive index 2.03 carved into taper or slab shapes. Metamaterials are made of U-shaped zinc coated iron staples embedded in a polystyrene foam slab. For the numerical calculations, we used the Finite-Difference Time-Domain Method. Dispersive dielectric materials, both normal and anomalous, are modeled as the Drude dispersive media and handled using the Auxiliary Differential Equation Method.

1. Macleod, H. A. Thin-Film Optical Filters. (Taylor \& Francis, 2009).

2. Raut, H. K., Ganesh, V. A., Nair, A. S. \& Ramakrishna, S. Anti-reflective coatings: a critical, in-depth review. Energy Environ. Sci. 4, 3779 (2011).

3. Chattopadhyay, S. et al. Anti-reflecting and photonic nanostructures. Mat. Sci. Eng. R. 69, 1-35 (2010). 
4. Rayleigh, L. On reflection of vibrations at the confines of two media between which the transition is gradual. Proc. London Math. Soc. s1-11, 51-56 (1879).

5. Clapham, P. B. \& Hutley, M. C. Reduction of lens reflexion by the "moth eye" principle. Nature 244, 281-282 (1973).

6. Zhmakin, A. I. Enhancement of light extraction from light emitting diodes. Phys. Rep. 498, 189-241 (2011).

7. Singh, R., Narayanan Unni, K. N. \& Solanki, A. Improving the contrast ratio of OLED displays: an analysis of various techniques. Opt. Mater. 34, 716-723 (2012).

8. Parida, B., Iniyan, S. \& Goic, R. A review of solar photovoltaic technologies. Renew. Sust. Energ. Rev. 15, 1625-1636 (2011).

9. Lee, C., Bae, S. Y., Mobasser, S. \& Manohara, H. A novel silicon nanotips antireflection surface for the micro Sun sensor. Nano Lett. 5, 2438-2442 (2005).

10. Yeh, P. Optical Waves in Layered Media. (Wiley, 2005).

11. Southwell, W. H. Gradient-index antireflection coatings. Opt. Lett. 8, 584-586 (1983).

12. Grann, E. B., Varga, M. G. \& Pommet, D. A. Optimal design for antireflective tapered two-dimensional subwavelength grating structures. J. Opt. Soc. Am. A 12, 333 (1995).

13. Wilson, S. J. \& Hutley, M. C. The optical properties of "moth eye" antireflection surfaces. Opt. Acta. 29, 993-1009 (1982).

14. Southwell, W. H. Pyramid-array surface-relief structures producing antireflection index matching on optical surfaces. J. Opt. Soc. Am. A 8, 549 (1991).

15. Klopfenstein, R. A transmission line taper of improved design. Proc. IRE 44, 31-35 (1956)

16. Pozar, D. M. Microwave Engineering. (Wiley, 1997).

17. Wang, L. J., Kuzmich, A. \& Dogariu, A. Gain-assisted superluminal light propagation. Nature 406, 277-279 (2000)
18. Chen, H.-T. et al. Antireflection coating using metamaterials and identification of its mechanism. Phys. Rev. Lett. 105, 1-4 (2010).

\section{Acknowledgments}

We thank Ji-Hun Kang for his help in preparing metamaterials. This work was supported by the National Research Foundation of Korea (NRF) grant funded by the Korea government (MEST) (No.2011-0029807) and the Green Nano Technology Development Program (2011-0020205)

\section{Author contributions}

Q.P. developed the theory and K.K. performed the microwave measurement and the numerical calculation. Q.P. and K.K. analysed the data and wrote the manuscript.

\section{Additional information}

Supplementary information accompanies this paper at http://www.nature.com/ scientificreports

Competing financial interests: The authors declare no competing financial interests.

License: This work is licensed under a Creative Commons

Attribution-NonCommercial-NoDerivs 3.0 Unported License. To view a copy of this license, visit http://creativecommons.org/licenses/by-nc-nd/3.0/

How to cite this article: Kim, K. \& Park, Q.-H. Perfect anti-reflection from first principles. Sci. Rep. 3, 1062; DOI:10.1038/srep01062 (2013). 